\title{
Radiofrequency ablation of an epicardial accessory pathway in 19-year-old male
}

Damian Łasocha ${ }^{1, \mathrm{~A}-\mathrm{F}}$, Andrzej Hasiec ${ }^{2, \mathrm{~B}-\mathrm{C}, \mathrm{E}}$, Katarzyna Kalin $^{2, \mathrm{~B}-\mathrm{C}}$, Maria Bilińska ${ }^{2, \mathrm{C}, \mathrm{E}}$, Łukasz Szumowski $^{2, \mathrm{~A}-\mathrm{C}, \mathrm{E}-\mathrm{F}}$

A - Research concept and design, B - Collection and/or assembly of data, C - Data analysis and interpretation,

D - Writing the article, E - Critical revision of the article, F - Final approval of article

1 Department of Arrhythmias, Institute of Cardiology, Warsaw, Poland

2 Institute of Cardiology, Warsaw, Poland

Address for correspondence:

Damian Łasocha, Institute of Cardiology, Warsaw, Poland

email: dlasocha@ikard.pl

Andrzej Hasiec, Institute of Cardiology, Warsaw, Poland

email: hasand@o2.pl

Katarzyna Kalin, Institute of Cardiology, Warsaw, Poland

email: katarzyna.kalin@gmail.com

Maria Bilińska, Institute of Cardiology, Warsaw, Poland

email: mbilinska@ikard.pl

Łukasz Szumowski, Institute of Cardiology, Warsaw, Poland

email: lszumowski@ikard.pl

Received: 27.12.2016

Revised: 28.12.2016

Accepted: 28.12.2016

\section{Key words:}

\section{Wolff-Parkinson-White syndrome, catheter ablation, epicardial mapping}

\section{Case Report}

A 19-year-old man with a history of palpitations and syncope was referred for Wolff-Parkinson-White syndrome (WPW) ablation. The patient had undergone a previous unsuccessful procedure. Since then, despite administration of beta-blockers he had experienced a few arrhythmic episodes terminated by Valsalva maneuvers.

The surface electrogram (ECG) showed a short PR interval, a positive delta wave in leads II, III, and aVF and a negative one in precordial leads V1 and V2 (Fig. 1A). During the
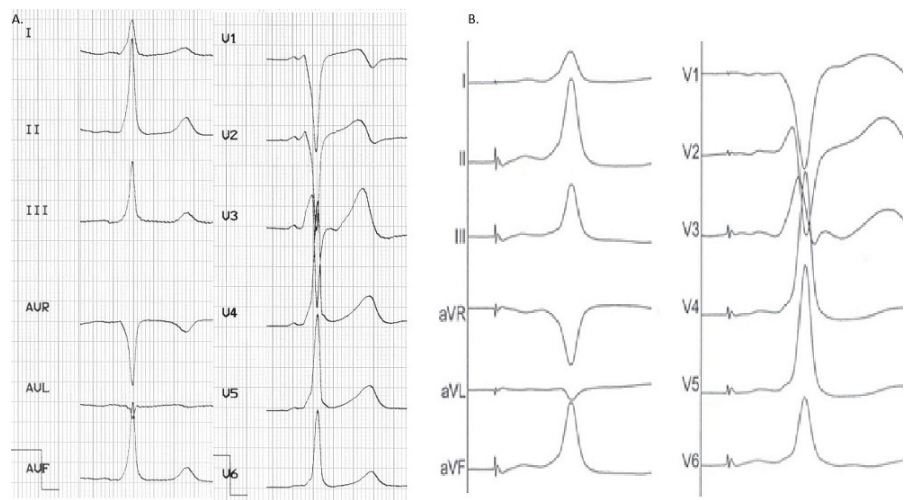

Figure $1 \mathrm{~A}$. Twelve-lead surface ECG showing delta wave. B. Maximal preexcitation during pacing. electrophysiological study maximal preexcitation confirmed the preexcitation pattern (Fig. 1B, 3A). An algorithm used to
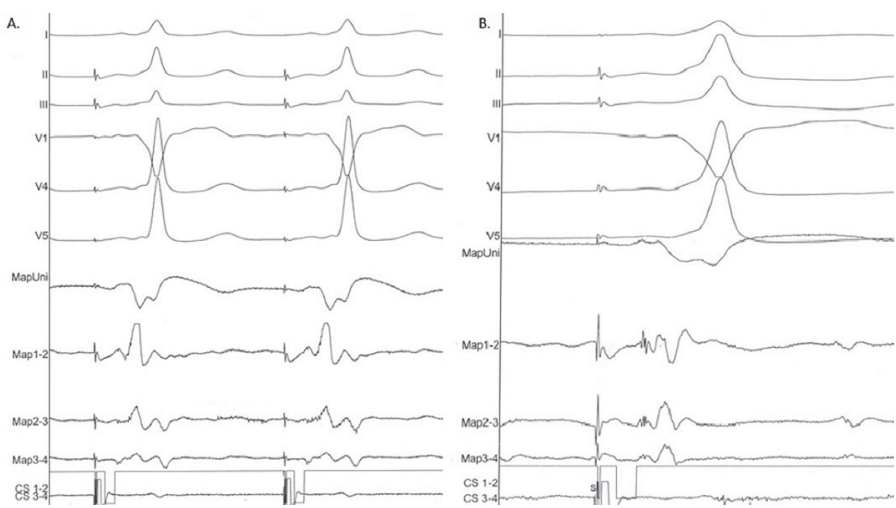

Figure 3 Comparison of endocardial (A) and epicardial (B) intracardiac signals.

localize accessory pathways suggested the right ventricle (RV) as the most likely ablation site (Fig. 2).

During the electrophysiological study (EP study) atrial fibrillation was induced, conducted by the accessory pathway (AP) with the shortest cycle length, reaching $220 \mathrm{~ms}$. Electrical cardioversion was performed to restore sinus rhythm.

Endocardial mapping using a 3D electroanatomical system indicated the earliest ventricular activation signal in right 


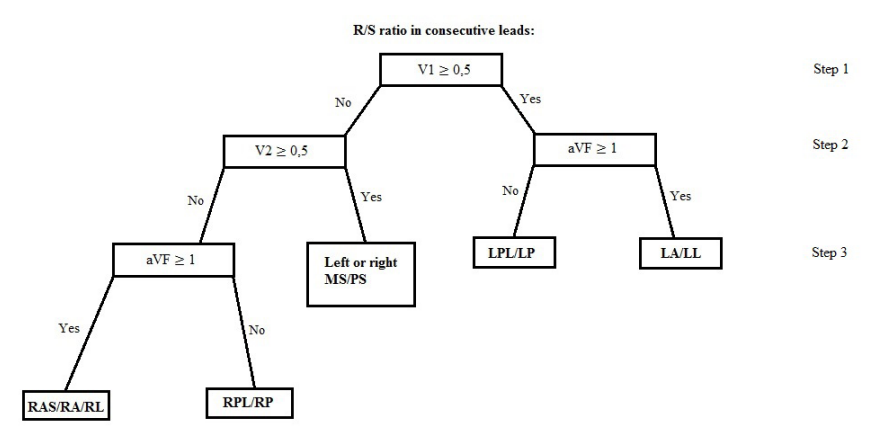

Figure 2 Accessory pathways' algorithm based on R/S ratio [1].
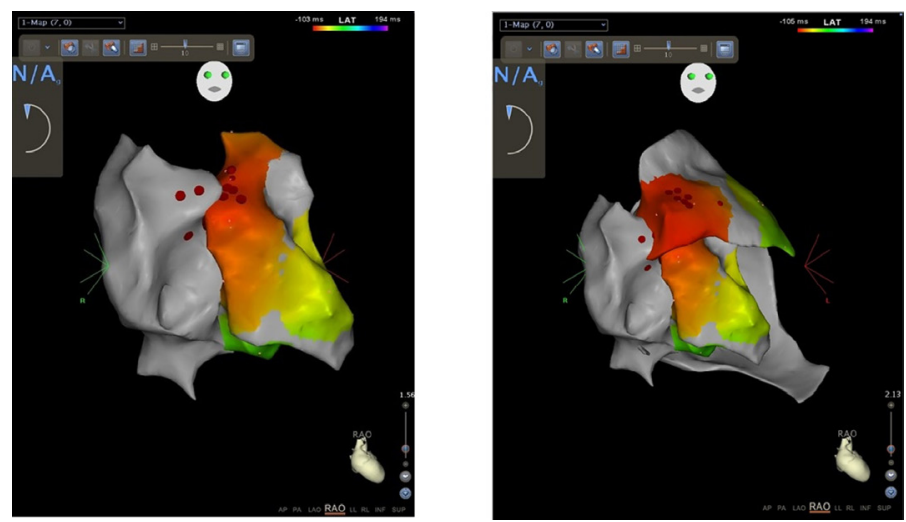

Figure 4 Left: Endocardial map with the earliest signals in RVOT. Right: Epicardial mapping. Note that both maps (endo- and epicardial) indicate the same area of early activation.

ventricular outflow tract (RVOT) (Fig. 4). The earliest atrioventricular transition signals were detected within the right atrial auricle (RAA). Although endocardial RF applications in this area removed AP conduction, preexcitation returned in the follow-up after previous procedures.

During the current ablation session endocardial RF applications in the area of the RVOT and RAA changed local AP conduction, but the delta wave remained.

After converting to the epicardial approach, coronarography was performed to assess right coronary artery (RCA) location (Fig. 5). Epicardial mapping combined with coronar-

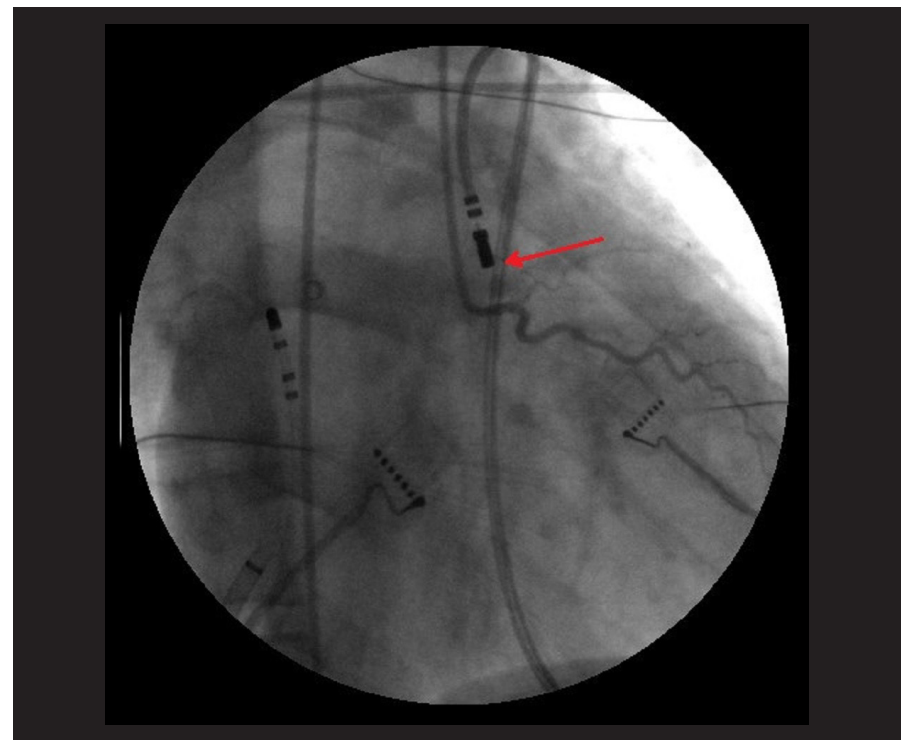

Figure 5 Coronary angiography of RCA. Ablation catheter's tip (arrow) shows successful application site. ography showed the earliest activation signal approximately $10 \mathrm{~mm}$ from the right coronary artery (RCA). RF applications delivered in this epicardial area successfully terminated AP conduction (Fig. 6). At 3- and 6-month follow-up there was neither clinical nor electrophysiological WPW recurrence.

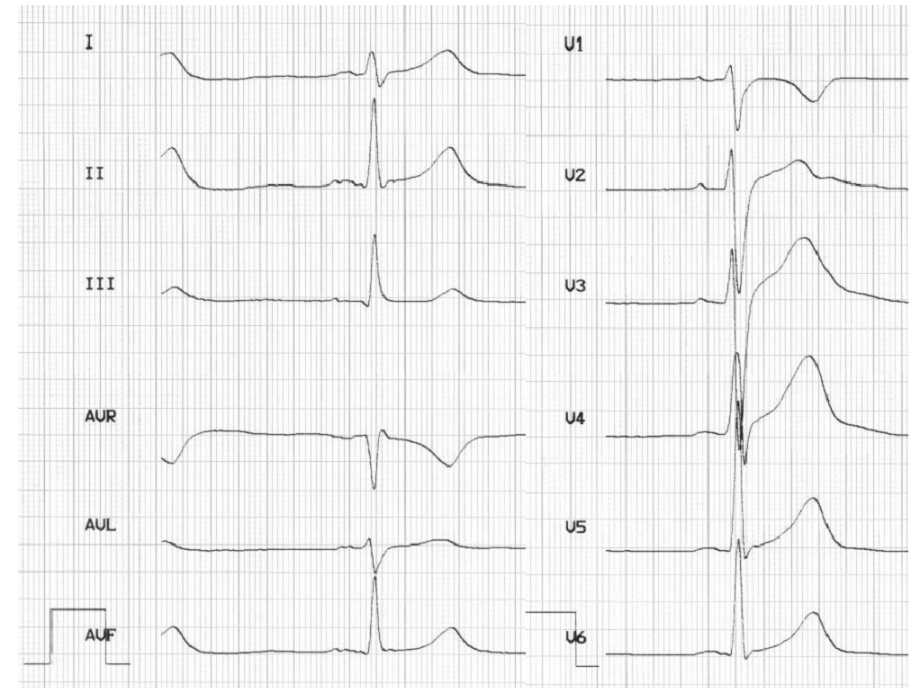

Figure 6 Disappearance of preexcitation after RF ablation in twelve-lead surface ECG.

\section{Discussion}

Catheter ablation of Wolff-Parkinson-White syndrome is a highly effective procedure with a low complication rate.

However, in atypical clinical scenarios it can be challenging and might be associated with procedural failure. The main causes of unsuccessful procedures are difficulties in catheter manipulation, AP mapping difficulties due to its localization (epicardial, parahisian), multiple ( $>3$ ) pathways and congenital heart diseases ${ }^{[2]}$.

Epicardial pathway location has been cited as a reason for failed endocardial ablation in $8 \%$ of cases ${ }^{[3]}$. Epicardial accessory pathways occur in multiple locations, but the most common ones are posteroseptal and left posterior accessory pathways connecting the coronary sinus (CS) with the left ventricle myocardium (19-33\% of epicardial APs). In this case successful ablation is usually performed within the CS or its branches. Most published reports focus on the above-mentioned location where the AP is located remotely from the endocardium, and such an AP is called 'epicardial'. In our opinion a 'deep' AP would be a better description, whereas 'epicardial' should be reserved for an AP connecting anatomical structures lying away from the tricuspid or mitral annulus. A rarely reported connection between the atrial appendage and right or left ventricle outflow tract would be an example. Termination of such an accessory pathway requires an epicardial approach [4].

In the current case previous endocardial procedures failed to remove the accessory pathway, in spite of multiple RF applications delivered at the RVOT and RAA. The epicardial approach resulted in a successful ablation.

Conclusion

In patients with WPW in whom surface ECG as well as the best mapping signals suggest ventricular AP insertion in the RVOT region an epicardial approach should be considered. 


\section{References}

1. Noriko T, Naoki Y, et al.: A simple algorithm for localizing accessory pathways in patients with Wolff-Parkinson-White syndrome using the R/S ratio. J of Arrhytmia 2014;30(6):439-443.

2. Sacher F, et al.: Wolff-Parkinson-White ablation after a prior failure: a 7-year multicentre experience. Europace. 2010;12:835.

3. Morady F, Strickberger A, Man KC, Daoud E, Niebauer M, Goyal R, Harvey M, Bogun F. Reasons for prolonged or failed attempts at radiofrequency catheter ablation of accessory pathways. Journal of the American College of Cardiology. 1996;27:683-689.

4. Sun Y, Arruda M, Otomo K, et al.: Coronary sinus-ventricular accessory connections producing posteroseptal and left posterior accessory pathways: Incidence and electrophysiological identification. Circulation. 2002;106:1362. 